\title{
Generalization of a Problem of Evelyn-Linfoot and Page in Additive Number Theory $\left.{ }^{*}\right)$.
}

\author{
D. Suryamarayana - V. Stta Ramatait (Waltair, India)
}

Summary. - Let $k$ and $l$ be integers such that $2 \leqslant k \leqslant l$. Let $S_{k}$ and $S_{l}^{\prime}$ be two subsets of positive integers with $S_{k} \supseteq Q_{k}$ and $S_{l}^{\prime} \supseteq Q_{l}$, where $Q_{k}$ denotes the set of k-free integers. Further suppose that the characteristic functions of $S_{k}$ and $S_{l}^{\prime}$ are multiplicative. Let $T(n)$ denote the number of representations of $n$ in the form $n=a+b$, where $a \in S_{k}$ and $b \in S_{l}^{I}$. In this paper we establish an asymptotic formula for $T(n)$, when $n$ is sufficiently large; and deduce several known asymptotic formulae as particular cases.

\section{1. - Introduction.}

Let $k$ and $l$ be two integers such that $2 \leqslant k \leqslant l$. Let $S_{l}$ and $S_{l}^{\prime}$ be two subsets of positive integers with $S_{k} \supseteq Q_{k}$ and $S_{l}^{\prime} \supseteq Q_{l}$, where $Q_{l k}$ denotes the set of $k$-free integers; that is, integers which are not divisible by the $k$-th power of any prime. Let $q_{k}$ denote the characteristic function of the set $Q_{k}$. Let $f_{k}$ and $f_{l}^{\prime}$ denote the characteristic functions of the sets $S_{k}$ and $S_{l}^{\prime}$; that is, $f_{k}(n)=1$ or 0 according as $n \in S_{k}^{\prime}$ or $n \notin S_{k}$ : We assume throughout that $f_{k}$ and $f_{l}^{\prime}$ are multiplicative. Define

and

$$
g_{k}(m)=\sum_{d ! m} \mu(d) f_{k}(m / d),
$$

where $\mu$ denotes the well-known Möbius function. Then by the converse of Möbius Inversion formula, we have

$$
f_{k}(m)=\sum_{d \mid m} g_{k}(d)
$$

and

$$
f_{l}^{\prime}(m)=\sum_{a \mid m} g_{l}^{\prime}(d)
$$

Let $T(n)=T\left(S_{k} ; S_{l}^{\prime}, n\right)$ denote the number of representations of $n$ in the form $n=a+b$, where $a \in S_{k}$ and $b \in S_{l}^{\prime}$. Then by (1.3) and (1.4) we have

$$
T(n)=\sum_{n=a+b} f_{k}(a) f_{l}^{\prime}(b)=\sum_{n=d_{1} \delta_{1}+d_{2} \delta_{3}} g_{k}\left(d_{1}\right) g_{l}^{\prime}\left(d_{2}\right) .
$$

(*) Entrata in Redazione il 7 maggio 1979.

1 - Annali di Matematica 
In this paper, we prove the following:

Theorem 1. - For sufficiently large $n$, we have

$$
\begin{aligned}
T(n)=n \cdot g(n) \cdot \prod_{p}\left\{\sum_{j=0}^{\infty} g_{l}^{\prime}\left(p^{j}\right) p^{-j}\right\} \cdot \prod_{p}\left\{1+\left(\sum_{j=0}^{\infty} g_{i}^{\prime}\left(p^{j}\right) p^{-j}\right)^{-1} \sum_{t=1}^{\infty} g_{k}(p t) p^{-t}\right\}+ \\
+O\left(n^{(k+l-2) /(k l-1)+s)},\right.
\end{aligned}
$$

for every $\varepsilon>0$, where the 0 -constant depends only on $k, l$ and $\varepsilon$,

$$
\begin{aligned}
S(n)=\prod_{\left.p\right|^{n}}\{1+ & \left.\left(\sum_{j=0}^{\infty} g_{l}^{\prime}\left(p^{j}\right) p^{-j}\right)^{-1} \sum_{t=1}^{\infty} g_{k}(p t) p^{-t}\right\}^{-1} \times \\
& \times \prod_{p \mid n}\left\{1+\left(\sum_{j=0}^{\infty} g_{l}^{\prime}\left(p^{j}\right) p^{-j}\right)^{-1} \cdot \sum_{t=1}^{\infty} g_{k}\left(p^{t}\right) p^{-t} \sum_{j=0}^{\infty} g_{l}^{\prime}\left(p^{j}\right) \varepsilon_{n}\left(\left(p^{j}, p^{t}\right)\right) p^{-j}\right\},
\end{aligned}
$$

and

$$
\varepsilon_{n}(m, b)= \begin{cases}(m, b), & \text { if }(m, b) \mid(n, b), \\ 0, & \text { otherwise. }\end{cases}
$$

Theorkim 2. - Let $S_{k}=Q_{k}$ and $T\left(Q_{k} ; S_{l}^{\prime}, n\right)=T_{k}\left(S_{l}^{\prime} ; n\right)$. Then for sufficiently large $n$, we have

$$
T_{k}\left(S_{l}^{\prime} ; n\right)=\frac{n}{\zeta(l)} \sum_{\substack{m=1 \\(m, n) \in Q_{k}}}^{\infty} \frac{g_{l}^{\prime}(m) m^{k-1}}{J_{k}(m)}+F_{k, l}(n)
$$

where $\zeta(k)$ is the Riemann zeta function defined by $\zeta(k)=\sum_{n=1}^{\infty} n^{-k}$,

$$
J_{k}(m)=m^{k} \prod_{p \mid m}\left(1-p^{-k}\right),
$$

the Jordan totient function, and for every $\varepsilon>0$,

$$
F_{k, l}(n)=O\left(n^{(k+l) /(k+1)+\varepsilon}\right) \quad \text { or } \quad O\left(n^{1 / k}\right),
$$

according as $l \leqslant k^{2}$ or $l>k^{2}$, where the $O$-estimates depend only on $k, l$ and $\varepsilon$.

THeorem 3. - When $l \leqslant k^{2}$, the error term $F_{k, l}(n)$ in formula (1.9) has the better $O$-estimate given by

$$
F_{k, l}(n)=O\left(n^{(k+l) / l(k+1)}(\log n)^{\left(k^{1 /(k+1)}-1\right)(l-1) / l}\right),
$$

where the $O$-constant depends only on $k, l$ and $\varepsilon$.

In section 2, we prepare the necessary background for proving Theorems 1,2 and 3 and in section 4, we give several applications of Theorems 1,2,3 and refer to the results that exist in the literature which are particular cases of these Theorems. 


\section{2. - Preliminaries.}

In this section, we prove some lemmas which are needed in our present discussion. Throughout the following $r$ denotes a fixed integer $\geqslant 2$.

LikMma 2.1 (cf. [15], Lemma 2.6). - Let $h_{r}$ be the multiplicative function defined by

$$
h_{r}\left(p^{\alpha}\right)= \begin{cases}0, & \text { if } 1 \leqslant \alpha \leqslant r-1 \\ 1, & \text { if } \alpha \geqslant r .\end{cases}
$$

Then $\sum_{m=1}^{\infty} h_{r}(m) m^{-}$converges for any $s>1 / r$.

LEMMA 2.2. - We have

$$
h_{r}(m)=\sum_{d^{r} \delta=m} h_{r}^{*}(\delta)
$$

where $h_{r}^{*}$ is a multiplicative function such that the series

$$
\sum_{m=1}^{\infty} h_{r}^{*}(m) m^{-s} \text { is convergent for } s>(r+1)^{-1} .
$$

Proof. - This is implicit in the proof of lemma 2.8 of [15].

Lema 2.3 (ef. [15], Lemma 2.8). - For $x \geqslant 1$,

$$
\sum_{m \leqslant x} h_{r}(m)=O\left(x^{1 / r}\right)
$$

where the $O$-constant depends only on $r$.

Lemma 2.4. - Let $m$ be a fixed positive integer. Then

$$
\sum_{n \leqslant s} h_{r}(m n)=O\left((x m)^{1 / r}\right)
$$

where the $O$-constant depends only on $r$.

Proof. - We have

$$
\sum_{n \leqslant x} h_{r}(m n)=\sum_{\substack{t \leqslant x m \\ m \mid t}} h_{r}(t) \leqslant \sum_{t \leqslant x m} h_{r}(t)=O\left((x m)^{1 / r}\right)
$$

by Lemma 2.3.

Hence Lemma 2.4 follows. 
LEMMA 2.5. - For each fixed $m$,

$$
\sum_{n>\infty} \frac{h_{r}(m n)}{n}=O\left(x^{-1+1 / r} \cdot m^{1 / r}\right)
$$

where the $O$-constant depends only on $r$.

Proof. - Follows from Lemma 2.4 and partial summation.

Lemna 2.6. - For each fixed $m$,

$$
\sum_{n=1}^{\infty} h_{r}(m n) n^{-1}=O\left(m^{\varepsilon}\right), \quad \text { for every } \varepsilon>0
$$

where the $O$-constant depends only on $\varepsilon$.

Proof. - We have

$$
\sum_{n \leqslant x} h_{r}(m n) n^{-1}=m \sum_{\substack{t \leqslant x m m \\ m \mid t}} h_{r}(t) t^{-1} \leqslant m \sum_{t \leqslant x m} h_{r}(t) t^{-1}
$$

Since the series $\sum_{t=1}^{\infty} h_{r}(t) t^{-1}$, converges by Lemma 2.1 , it follows that the series $\sum_{n=1}^{\infty} h_{r}(m n) n^{-1}$ also converges.

We have

$$
\begin{aligned}
\sum_{n=1}^{\infty} h_{r}(m n) n^{-1} & =\left(\sum_{\substack{n_{1}=1 \\
\left(n_{1}, m\right)=1}}^{\infty} h_{r}\left(n_{1}\right) n_{1}^{-1}\right) \prod_{p^{\alpha} \mid m n}\left\{h_{r}\left(p^{\alpha}\right)+\frac{h_{r}\left(p^{\alpha+1}\right)}{p}+\ldots\right\}= \\
& =\prod_{p \nmid m}\left\{1+\frac{1}{p^{r}}+\frac{1}{p^{r+1}}+\ldots\right\} \prod_{p^{\alpha} \mid m m}\left\{h_{r}\left(p^{\alpha}\right)+\frac{h_{r}\left(p^{\alpha+1}\right)}{p}+\ldots\right\}< \\
& <\prod_{p}\left\{1+\frac{1}{p^{r}}+\frac{1}{p^{r+1}}+\ldots\right\} \prod_{p ! m}\left\{1+\frac{1}{p}+\frac{1}{p^{2}}+\ldots\right\} \leqslant \zeta(2) \cdot 2 \omega(m)=O\left(m^{\varepsilon}\right),
\end{aligned}
$$

where $\omega(m)$ is the number of distinct prime factors of $m$.

Hence lemma 2.6 follows.

LEMMA 2.7. - If $g_{k}, g_{l}^{\prime}$ and $h_{r}$ are the multiplicative functions defined in (1.1), (1.2) and (2.1), then

$$
\left|g_{k}(m)\right| \leqslant h_{k}(m)
$$

and

$$
\left|g_{l}^{\prime}(m)\right| \leqslant h_{l}(m),
$$

for all $m$. 
PRoof. - The proof of (2.4) is given in Lemma 2.14 of [15]. (2.5) similarly follows.

LEMMA 2.8 (cf. [9], Lemma 2.1, p. 132). - If $a, b, n$ are positive integers and $R(a$, $b, n)$ denotes the numbers of solutions in positive integers $x, y$ of the Diophantine equation

$$
a x+b y=n,
$$

then

$$
R(a, b, n)= \begin{cases}\frac{n(a, b)}{a b}+2 \theta_{1}, & \text { if } a+b \leqslant n,(a, b) \mid n \\ 0, \quad \text { otherwise, } & \end{cases}
$$

where $\theta_{1}=\theta_{1}(a, b, n)$ is a number absolutely less than 1 .

For positive integers $a$ and $H$, let $Q_{k}(x ; \alpha, H)$ denote the number of positive integers $n \leqslant x$ with $n \equiv a(\bmod H)$. As usual, $H$ is called $K$-full if $p^{\bar{k}} \mid H$ for any prime $p \mid H$.

LEIDYA 2.9. - If $(a, H) \in Q_{k}$ and $H$ is $k$-full, then

$$
Q_{k}(x ; \alpha, H)=\frac{x H^{k-1}}{\zeta(k) J_{k}(H)}+E_{k}(x ; a, H),
$$

where

$$
E_{k}(x ; a, H)=O\left(k^{\omega(H)}\left(x^{1 / k} H^{-1 / k^{2}}+H^{1 / k}\right)\right)
$$

the $O$-estimates being uniform in $x, a$ and $H ; J_{l}(H)$ being given by $(1.10)$.

REMARK 2.1. - K. PrachaR (ef. [14], p. 175) proved (2.6) under the assumption $(a, H)=1$, rather than the assumption $(a, H) \in Q_{k}$ and $H$ is $k$-full. However, the proof in either case is almost identical (cf. [14], §3). Infact, C. Pomerence and D. SURYANARAYANA [13] recently made small improvements in the $O$-estimates of (2.7) even under less stringent conditions on and $H$.

LEMMA 2.10 (cf. [13], corollary). - We have

$$
E_{k}(x ; a, H)= \begin{cases}O\left(\left(x k^{\omega(H)}\right)^{1 / k+1}\right), & \text { if } x \leqslant H^{k} \\ O\left(x^{1 / k} H^{-1 /(k+3 / 2)}\right), & \text { if } x>H^{k},\end{cases}
$$

where the implied constants in the $O$-terms are uniform in $x$, an and $H$.

REMARK 2.2. - It is clear that $Q_{k}(x ; a, H)=0$, if $(a, H) \notin Q_{k}$. Further, if $Q_{k}^{*}(x$; $a, H)$ denotes the number of positive integers strictly less than $x$ with $n \equiv a(\bmod H)$, then $Q_{k}(x ; a, H)$ and $Q_{k}^{*}(x ; a, H)$ differ by atmost 1 so that

$$
Q_{k}^{*}(x ; a, H)=Q_{k}(x ; a, H)+O(1)
$$




\section{3. - Main results.}

In this section we give the proofs of Theorems 1,2 and 3 stated in the introduction.

Proof of THEOREM 1. - We give the proof of this theorem by combining the methods due to CARLItz [2] and PAge [12]. Liet $1<t_{i}=t_{i}(n)<n, i=1,2$, be two functions of $n$. By (1.5), we have

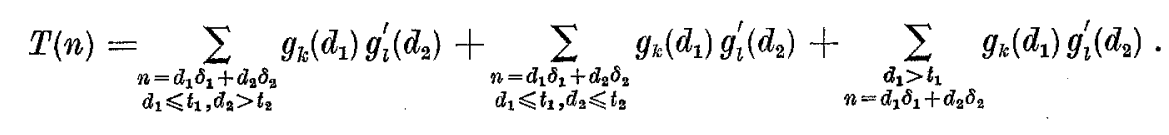

Let

$$
T^{\prime}(n)=\sum_{\substack{n=d_{1} \delta_{1}+d_{2} \delta_{0} \delta_{2} \\ d_{1} \leqslant t_{1}, d_{2} \leqslant t_{2}}} g_{k}\left(d_{1}\right) g_{b}^{\prime}\left(d_{2}\right)
$$

so that by $(2.4)$ and $(2.5)$, we have

$$
\left|T(n)-T^{\prime}(n)\right|<\sum_{\substack{n=d_{1} \delta_{1}+d_{2} \delta_{2} \\ d_{2}>t_{2}}} h_{k}\left(d_{1}\right) h_{l}\left(d_{2}\right)+\sum_{\substack{n=d_{1} \delta_{1}+d_{2} \delta_{2} \\ d_{1}>t_{1}}} h_{k}\left(d_{1}\right) h_{l}\left(d_{2}\right)=T_{2}+T_{1}, \quad \text { say. }
$$

By Lemma $2.5(m=1, r=l)$ we have

$$
\begin{array}{r}
T_{2} \leqslant \sum_{\substack{d_{2} \delta_{2}<n \\
d_{2}>t_{2}}} h_{l}\left(d_{2}\right) \sum_{d_{1} \mid n-d_{2} \delta_{2}} 1=\sum_{\substack{d_{2} \delta_{2}<n \\
d_{3}>t_{2}}} h_{l}\left(d_{2}\right) \tau\left(n-d_{2} \delta_{2}\right)=O\left(n^{\varepsilon} \sum_{\substack{d_{2}<n \\
d_{2}>t_{2}}} h_{l}\left(d_{2}\right) \sum_{\delta_{2}<n / d_{2}} 1\right)= \\
=O\left(n^{1+\varepsilon} \sum_{d_{2}>t_{2}} h_{l}\left(d_{2}\right) d_{2}^{-1}\right)=O\left(n^{1+\varepsilon} t_{2}^{-1+1 / l}\right),
\end{array}
$$

where in the above, we used that $\tau(n)=O\left(n^{\varepsilon}\right)$ for every $\varepsilon>0, \tau(n)$ being the number of divisors of $n$.

Similarly $T_{1}=O\left(n^{1+\varepsilon} t_{1}^{-1+1 / k}\right)$. Hence we have

$$
T(n)=T^{\prime}(n)+O\left(n^{1+\varepsilon} t_{2}^{-1+1 / l}\right)+O\left(n^{1+\varepsilon} t_{1}^{-1+1 / k}\right) .
$$

From (3.1), we have

$$
T^{\prime}(n) \leqslant \sum_{\substack{m \mid n \\ m}} \sum_{\substack{n=d_{1} \delta_{1}+d_{2} \delta_{2} \\ d_{1} \leq t_{1}, d_{3} \leq t_{2} \\\left(d_{1}, d_{3}\right)=m}} g_{k}\left(d_{1}\right) g_{l}^{\prime}\left(d_{2}\right)=\sum_{m \mid n} T^{*}(m), \quad \text { say. }
$$


Now, by lemma 2.8,

$$
\begin{gathered}
T^{*}(m)=\sum_{\substack{n=d_{8} \delta_{1}+d_{2} \delta_{2} \\
d_{1} \leqslant t_{1}, d_{2} \leqslant t_{2} \\
\left(d_{1}, d_{2}\right)=m}} g_{k}\left(d_{1}\right) g_{l}^{\prime}\left(d_{2}\right)=\sum_{\substack{n / m=a_{1} \delta_{1}+a_{2} \delta_{2} \\
\left(a_{1}, a_{2}\right)=1 \\
a_{1} \leqslant t_{1} / m, a_{2} \leqslant t_{2} / m}} g_{k}\left(a_{1} m\right) g_{l}^{\prime}\left(a_{2} m\right)= \\
=\sum_{\substack{a_{1} \leqslant t_{1} / m \\
a_{2} \leqslant t_{2} / m \\
\left(a_{1}, a_{2}\right)=1}} g_{k}\left(a_{1} m\right) g_{l}^{\prime}\left(a_{2} m\right)\left\{\frac{n}{m a_{1} a_{2}}+O(1)\right\}= \\
=n m^{-1} \sum_{\substack{a_{1} \leqslant t_{1} / m \\
a_{2} \leqslant t_{3} / m \\
\left(a_{1}, a_{2}\right)=1}} g_{k}\left(a_{1} m\right) g_{l}^{\prime}\left(a_{2} m\right) / a_{1} a_{2}+O\left(\sum_{\substack{a_{1} \leqslant t_{1} / m \\
a_{2} \leqslant t_{2} / m}} h_{k}\left(a_{1} m\right) h_{l}\left(a_{2} m\right)\right) .
\end{gathered}
$$

By lemma 2.3, the $O$-term in $(3.4)$ is $O\left(t_{1}^{1 / k} t_{2}^{1 / h}\right)$, so that

$$
\begin{aligned}
& \quad T^{*}(m)=n m^{-1} \sum_{\substack{a_{1} \leqslant t_{1} / m \\
a_{2}<a_{2} / m \\
\left(a_{1}, a_{2}\right)=1}} g_{l}\left(a_{1} m\right) g_{l}^{\prime}\left(a_{2} m\right) / a_{1} a_{2}+O\left(t_{1}^{1 / k} t_{2}^{1 / l}\right)= \\
& =n m^{-1} \sum_{a_{1} \leqslant t_{1} / m} g_{k}\left(a_{1} m\right) a_{1}^{-1}\left\{\sum_{\substack{a_{2}=1 \\
\left(a_{2}, a_{1}\right)=1}}^{\infty} g_{l}^{\prime}\left(a_{2} m\right) a_{2}^{-1}+O\left(\sum_{a_{2}>t_{2} / m} h_{l}\left(a_{2} m\right) a_{2}^{-1}\right)\right\}+O\left(t_{1}^{1 / k} t_{2}^{1 / l}\right)= \\
& =n m^{-1} \sum_{a_{1} \leqslant t_{1} / m} g_{k}\left(a_{1} m\right) A_{l}^{\prime}\left(a_{1} ; m\right) a_{1}^{-1}+O\left(t_{2}^{-1+1 / l} n \sum_{a_{1} \leqslant t_{1} / m} h_{k}\left(a_{1} m\right) / a_{1}\right)+O\left(t_{1}^{1 / k} t_{2}^{1 / l}\right) .
\end{aligned}
$$

by Lemma 2.5 , where

$$
A_{l}^{\prime}\left(a_{1} ; m\right)=\sum_{\substack{a_{2}=1 \\\left(a_{2}, a_{1}\right)=1}}^{\infty} g_{l}^{\prime}\left(a_{2} m\right) a_{1}^{-1}
$$

Now, by Lemma 2.6,

$$
\sum_{a_{1} \leqslant t_{1} / m} h_{k}\left(a_{1} m\right) a_{1}^{-1} \leqslant \sum_{a_{1}=1}^{\infty} h_{k}\left(a_{1} m\right) a_{1}^{-1}=O\left(m^{\varepsilon}\right) .
$$

Further, we have

$$
\begin{aligned}
T_{3}=\sum_{a_{1} \leqslant t_{1} / m} g_{k}\left(a_{1} m\right) A_{l}^{\prime}\left(a_{1} ; m\right) a_{1}^{-1}=\sum_{a_{1}=1}^{\infty} g_{k}\left(a_{1} m\right) A_{l}^{\prime}\left(a_{1} ; m\right) a_{1}^{-1} & + \\
& +O\left(\sum_{a_{1}>t_{1} / m} h_{k}\left(a_{1} m\right) A_{l}^{\prime}\left(a_{1} ; m\right) a_{1}^{-1}\right) .
\end{aligned}
$$

Since by Lemma 2.6, $A_{l}^{\prime}\left(a_{1} ; m\right) \leqslant \sum_{a_{2}=1}^{\infty} h_{l}\left(a_{2} m\right) a_{2}^{-1}=O\left(m^{8}\right)$, it follows from Lemma 2.5 $(r=k)$ that

$$
T_{\mathbf{3}}=\sum_{a_{1}=1}^{\infty} g_{k}\left(a_{1} m\right) A_{l}^{\prime}\left(a_{1} ; m\right) a_{1}^{-1}+O\left(t_{1}^{-1+1 / k} m^{1+\varepsilon}\right)
$$


Collecting (3.5)-(3.8), we have

$$
\begin{aligned}
T^{*}(m)=n m^{-1} \sum_{a_{1}=1}^{\infty} g_{l}\left(a_{1} m\right) A_{l}^{\prime}\left(a_{1} ; m\right) & a_{1}^{-1}+ \\
& +O\left(t_{1}^{-1+1 / k} m^{1+\varepsilon}\right)+O\left(t_{2}^{-1+1 / l} n m^{\varepsilon}\right)+O\left(t_{1}^{1 / k} t_{2}^{1 / l}\right),
\end{aligned}
$$

so that

$$
\begin{aligned}
T^{\prime}(n)=\sum_{m \mid n} T^{*}(m)=n \sum_{m \mid n} m^{-1} \sum_{a_{1}=1}^{\infty} g_{k}\left(a_{1} m\right) A_{l}^{\prime}\left(a_{1} ; m\right) a_{1}^{-1}+ \\
\quad+O\left(t_{1}^{-1+1 / k} \sum_{m \mid n} m^{1+\varepsilon}\right)+O\left(n t_{2}^{-1+1 / l} \sum_{m \mid n} m^{\varepsilon}\right)+O\left(t_{1}^{1 / k} t_{2}^{1 / h} \tau(n)\right) .
\end{aligned}
$$

It is elear that $\sum_{m \mid n} m^{1+\varepsilon} \leqslant n^{1+\varepsilon} \cdot \tau(n)=O\left(n^{1+2 \varepsilon}\right)$, and $\sum_{m \mid \varepsilon} m^{\varepsilon} \leqslant n^{\varepsilon} \tau(n)=O\left(n^{2 \varepsilon}\right)$, for every $\varepsilon>0$. Hence we have

$$
T^{\prime}(n)=n \sum_{m \mid n} H(m) m^{-1}+O\left(n^{1+2 \varepsilon} t_{1}^{-1+1 / k}\right)+O\left(n^{1+2 \varepsilon} t_{2}^{-1+1 / l}\right)+O\left(t_{1}^{1 / k} t_{2}^{1 / l} n^{\varepsilon}\right),
$$

where $H(m)=\sum_{a_{1}=1}^{\infty} g_{k}\left(a_{1} m\right) A_{l}^{\prime}\left(a_{1} ; m\right) a_{1}^{-1}$. Now by $(3.3)$, it follows that

$$
T(n)=n \sum_{m \mid / n} H(m) m^{-1}+O\left(n^{1+2 \varepsilon} t_{1}^{-1+1 / k}\right)+O\left(n^{1+2 \varepsilon} t_{2}^{-1+1 / h}\right)+O\left(n^{\varepsilon} t_{1}^{1 / k} t_{2}^{1 / k}\right) .
$$

Taking $t_{1}=n^{k(l-1) /(k l-1)}$ and $t_{2}=n^{l(k-1) /(k l-1)}$, we see that each of the above $O$-terms reduce to $O\left(n^{(k+l-2) /(k l-1)+26}\right)$. Now, it can be shown using the Euler- Infinite Product theorem that

$$
\begin{aligned}
\sum_{m \mid n} H(m) m^{-1}=\sum_{\substack{a_{1}, a_{3}=1 \\
\left(a_{2}, a_{2}\right) \mid n}}^{\infty} g_{k}\left(a_{1}\right) g_{l}^{\prime}\left(a_{2}\right)\left(a_{1}, a_{2}\right)\left(a_{1} a_{2}\right)^{-1}= \\
=S(n) \cdot \prod_{p}\left\{\sum_{j=0}^{\infty} g_{l}^{\prime}\left(p^{j}\right) p^{-j}\right\} \prod_{p}\left\{1+\left(\sum_{j=0}^{\infty} g_{i}^{\prime}\left(p^{j}\right) p^{-j}\right)^{-1} \sum_{t=1}^{\infty} g_{k}(p t) p^{-i}\right\},
\end{aligned}
$$

where $S(n)$ is given by. (1.7).

Hence Theorem 1 follows.

REMARK 3.1. - We can further simplify the main term in (1.6) so that $T(n)$ is also given by

$$
\begin{aligned}
& T(n)=n \prod_{p}\left\{\sum_{j=0}^{\infty} g_{l}^{\prime}\left(p^{j}\right) p^{-j}+\sum_{i=1}^{\infty} g_{k}(p t) p^{-t}\right\} \prod_{p \mid n}\left\{\sum_{j=0}^{\infty} g_{l}^{\prime}\left(p^{j}\right) p^{-j}+\sum_{t=1}^{\infty} g_{k}(p t) p^{-t}\right\}^{-1} \times \\
& \times \prod_{p \mid n}\left\{\sum_{j=0}^{\infty} g_{l}^{\prime}\left(p^{j}\right) p^{-j}+\sum_{t=1}^{\infty} g_{k}\left(p^{t}\right) p^{-t} \sum_{j=0}^{\infty} g_{l}^{\prime}\left(p^{j}\right) p^{-j} \varepsilon_{n}\left(\left(p^{j}, p^{t}\right)\right\}\right\}+O\left(n^{(k+l-2) /(k l-1)+\varepsilon}\right) .
\end{aligned}
$$


Proof of Theorem 2. - By (1.3), Remark 2.2 and Lemma 2.3, we have

$$
\begin{gathered}
T\left(B_{l}^{\prime} ; n\right)=\sum_{n=a+b} f_{l}^{\prime}(a) q_{k}(b)=\sum_{n=d \delta+b} g_{l}^{\prime}(d) q_{k}(b)= \\
=\sum_{d<n} g_{l}^{\prime}(d) Q_{k}^{*}(n ; n, d)=\sum_{d<n} g_{l}^{\prime}(d) Q_{k}(n ; n, d)+O\left(\sum_{d<n} h_{l}(d)\right)= \\
=\sum_{d<n} g_{l}^{\prime}(d) Q_{k}(n ; n, d)+O\left(n^{1 / l}\right) .
\end{gathered}
$$

Let $\mathbf{1}^{\prime}<t=t(n)<n$. Then by Remark 2.2,

$$
\begin{aligned}
& \sum_{d<n} g_{l}^{\prime}(d) Q_{k}(n ; n, d)=\sum_{d<n} g_{l}^{\prime}(d) q_{k}((d, n)) Q_{k}(n ; n, d)= \\
= & \sum_{d \leqslant t} g_{l}^{\prime}(d) q_{k}((d, n)) Q_{l}(n ; n, d)+\sum_{t<d<n} g_{l}^{\prime}(d) q_{k}((d, n)) Q_{k}(n ; n, d)=T_{4}+T_{5}, \quad \text { say. }
\end{aligned}
$$

Since $Q_{k}(n ; n, d) \leqslant 2 n d^{-1}$, it follows from Lemma $2.5(r=l, m=1)$ that

$$
\left|T_{5}\right| \leqslant 2 n \sum_{d>t} h_{l}(d) d^{-1}=O\left(n t^{-1+1 / 2}\right)
$$

From (2.5) and (2.1) $(r=l)$ it follows that $g_{l}^{\prime}\left(p^{\alpha}\right)=0$ for any prime $p$ and $1 \leqslant \alpha \leqslant$ $\leqslant l-1$. Hence we can assume that $d$ in the sum $T_{4}$ is $l$-full and hence $k$-full since $l \geqslant k$. Hence by Lemma 2.9, we have

$$
\begin{aligned}
T_{4}=\sum_{d \leqslant t} g_{l}^{\prime}(d) q_{k}((d, n))\left\{\frac{n d^{k-1}}{\zeta(k) J_{k}(d)}+O\left(n^{1 / k} k^{\omega(d)} d^{-1 / k^{2}}\right)+O\left(k^{\omega(d)} d^{1 / k}\right)\right\}= \\
=\frac{n}{\zeta(k)} \sum_{d \leqslant t} \frac{g_{l}^{\prime}(d) q_{k}((d, n)) d^{k-1}}{J_{k}(d)}+O\left(n^{1 / k} \sum_{d \leqslant t} h_{l}(d) k^{\omega(d)} d^{-1 / k^{2}}\right)+ \\
+O\left(\sum_{d \leqslant t} h_{l}(d) k^{\omega(d)} d^{1 / k}\right)=T_{11}+T_{12}+T_{13}, \quad \text { say. }
\end{aligned}
$$

The series $\sum_{d=1}^{\infty} g_{l}^{\prime}(d) q_{k}((d, n)) d^{k-1} / J_{k}(d)$ converges absolutely by $(2.5)$, the fact that $d^{k} / J_{k}(d)$ is bounded for all $d$ and Lemma 2.1. Further by $(2.5)$ and Lemma $2.5(m=1$, $r=l)$,

$$
\sum_{d>t} g_{l}^{\prime}(d) q_{k}((d, n)) d^{k-1} / J_{k}(d)=O\left(\sum_{d>t} h_{l}(d) d^{-1}\right)=O\left(t^{-1+1 / l}\right)
$$

so that

$$
T_{11}=n(\zeta(k))^{-1} \sum_{d=1}^{\infty} g_{l}^{\prime}(d) q_{k}((d, n)) d^{k-1} / J_{l k}(d)+O\left(n t^{-1+1 / l}\right)
$$

By Lemmas 2.1 and 2.3 , and the fact that $k^{\omega(d)}=O\left(d^{\varepsilon}\right)$ for every $\varepsilon>0$ and partial 
summation, we have

$$
T_{12}= \begin{cases}O\left(n^{1 / k}\right), & \text { if } l>k^{2} \\ O\left(n^{1 / k} t^{1 / l-1 / k^{2}+\varepsilon}\right), & \text { if } l \leqslant k^{2}\end{cases}
$$

Also, by Lemma 2.3,

$$
T_{13}=O\left(t^{1 / \hbar+\varepsilon} \sum_{d \leqslant t} h_{l}(d)\right)=O\left(t^{1 / l+1 / h+s}\right)
$$

Collecting now the results (3.10)-(3.16) we obtain,

$$
T\left(S_{l}^{\prime} ; n\right)=n(\zeta(k))^{-1} \sum_{d=1}^{\infty} g_{l}^{\prime}(d) q_{k}((d, n)) d^{k-1} / J_{k}(d)+F_{k, l}(n)
$$

where

$$
F_{k, l}(n)=O\left(n^{1 / l}\right)+O\left(n t^{-1+1 / l}\right)+O\left(t^{1 / l+1 / k+\varepsilon}\right)+ \begin{cases}O\left(n^{1 / k}\right), & \text { if } l>k^{2} \\ O\left(n^{1 / k} t^{1 / l-1 / k^{2}+\varepsilon}\right), & \text { if } l \leqslant k^{2} .\end{cases}
$$

Taking $t=n^{k /(k+1)}$ we obtain Theorem 2 .

Proof of Theorem 3. - From (3.11), we have

$$
\begin{aligned}
T_{4}= & \sum_{d<t} g_{l}^{\prime}(d) q_{k}((d, n)) Q_{k}(n ; n, d)= \\
=n(\zeta(k))^{-1} \sum_{d \leqslant t} g_{l}^{\prime}(d) d^{k-1} q_{l k}((d, n)) / J_{k}(d) & +\sum_{d<n^{1 / k}} g_{l}^{\prime}(d) q_{k}((d, n)) E_{l k}(n ; n, d)+ \\
& +\sum_{n^{1 / k} \leqslant d \leqslant t} g_{l}^{\prime}(d) q_{k}((d, n)) E_{k}(n ; n, d)= \\
= &
\end{aligned}
$$

Where $E_{k}(x ; a, H)$ is the error term in the formula (2.6).

By (2.5), Lemma 2.10, Lemmas 2.1 and 2.3, and partial summation,

$$
\begin{aligned}
T_{12}^{\prime} & =O\left(n^{1 / k} \sum_{d<n^{1 / k}} h_{l}(d) d^{-1 /(k+3 / 2)}\right)= \begin{cases}O\left(n^{1 / k}\right), & \text { if } l \geqslant k+2 \\
O\left(n^{1 / k+1 / k l-1 / k(k+3 / 2)}\right), & \text { if } l<k+2 .\end{cases} \\
& =O\left(n^{(k+l) /(k+1)}\right) .
\end{aligned}
$$

Again by (2.5) and lemma 2.10, we have

$$
\begin{aligned}
T_{13}^{\prime} & =O\left(\sum_{d<t} h_{l}(d)\left(n k^{\omega(d)}\right)^{1 /(k+1)}\right) \\
& =O\left(n^{1 /(k+1)} \sum_{d<t} h_{l}(d)\left(k^{\omega(d)}\right)^{1 /(k+1)}\right) .
\end{aligned}
$$


Now by (2.2) and (2.3),

$$
\begin{aligned}
\sum_{m \leqslant t} h_{l}(m)\left(k^{\omega(m)}\right)^{1 /(k+1)} & \leqslant \sum_{d^{\delta} \delta \leqslant t} h_{l}^{*}(\delta)\left(k^{\omega(\delta)}\right)^{1 /(k+1)}\left(k^{\omega(d)}\right)^{1 /(k+1)} \\
& =\sum_{\delta \leqslant t} h_{l}^{*}(\delta)\left(k^{\omega(\delta)}\right)^{1 /(k+1)} \sum_{d \leqslant(l / \delta)^{1 / l}}(\tau(d))^{\log l_{2} l^{1 /(k+1)}} \\
& =O\left(t^{1 / l}(\log n)^{\left(k^{1 / 1 /(k+1)}-1\right)} \sum_{\delta \leqslant t} h_{l}^{*}(\delta) \delta^{-1 / l}\left(k^{\omega(\delta)}\right)^{1 /(k+1)}\right) \\
& =O\left(t^{1 / l}(\log n)^{\left(k^{1 /(k+1)}-1\right)}\right),
\end{aligned}
$$

where we use a formula of Ramanujan (see Wilson [19], eq. (2.39)). Hence we have

$$
T_{13}^{\prime}=O\left(n^{1 /(k+1)} t^{1 / l}(\log n)^{\left(k^{1 /(k+1)}-1\right)}\right) .
$$

Collecting now the results (3.10)-(3.12), (3.14) and (3.16)-(3.18), we obtain

$$
F_{k, l}(n)=O\left(n t^{-1+1 / l}\right)+O\left(n^{(k+l) l(k+1)}\right)+O\left(n^{1 /(k+1)} t^{1 / l}(\log n)^{\left(k^{1 /(k+1)}-1\right)}\right) .
$$

Taking $t=n^{k /(k+1)}(\log n)^{-\left(k^{1 /(k+1)}-1\right)}$, we obtain Theorem 3 .

\section{4. - Applications.}

Throughout the following $r_{1}$ and $r_{2}$ denote integers with $2 \leqslant r_{1} \leqslant r_{2}$ and $k_{1}$ and $k_{2}$ denote integers with $k_{1}>r_{1}$ and $k_{2}>r_{2}$. A positive integer $m$ is called Semi-k-free [18] if in the canonical factorization of $m$, no exponent is equal to $k$. A positive integer $m$ is called unitarily $k$-free [3] if no exponent in the canonical factorization of $m$ is a multiple of $k$ and $m$ is called a $k$-skew integer of rank $t$, where $t$ is any positive integer, if in the canonical factorization of $m$, no exponent is equal to $j k$, for $1 \leqslant j \leqslant t$. For convenience, we shall call these integers ( $k$-skew integers of rank $t$ ) as unitarily $k-t$ integers. Clearly the unitarily $k-1$-integers are the semi-k-free integers and the unitarily $k-\infty$-integers are the unitarily $k$-free integers. Let $r$ be an integer with $2 \leqslant r<k$. A positive integer $m$ is called a $(k, r)$-free integer if no exponent in the canonical factorization of $m$ belongs to the interval $[r, k-1]$. This definition includes as special cases the $r$-free integers $(k=\infty)$ and the Semi-r $r$ free integers $(k=r+1)$. A positive integer $m$ is called a $(k, r)$-integer if $m$ can be represented as $m=m_{1}^{k} m_{2}$ where $m_{1}$ is a positive integer and $m_{2}$ is an $r$-free integer. These integers include as a special case the $r$-free integers $(k=\infty)$. The concept of a $(k, r)$-integer has been introduced independently (without using this name) by E. CoHEN [5] and M. V. SUBBa RuA - V. C. HarRIs [16]. Let $S_{t}^{*}$ denote the set of all integers $m$ in whose canonical factorization each exponent is just 1 or $t$. We also include the integer 1 in the set $S_{i}^{*}$. These integers have been introduced by E. CoHEN (cf. [4], p. 78). 
Let $Q_{k, t}^{*}, Q_{(k, r)}$ and $Q_{k, r}$ respectively denote the set of all unitarily $k-t,(k, r)$-free and $(k, r)$-integers. Let $q_{k, t}^{*}, q_{(k, r)}$ and $q_{k, r}$ denote the characteristic functions of the sets $Q_{k, t}^{*}, Q_{(k, r)}$ and $Q_{k, r}$ : First we have

COROLIARY 4.1. - Let $T_{\left(k_{1}, r_{1}\right)\left(k_{3}, r_{2}\right)}(n)$ denote the number of ordered pairs $(a, b) \in$ $\in Q_{k_{1}, r_{1}} \times Q_{k_{2}, r_{3}}$ with $n=a+b$. Then for sufficiently large $n$, we have

$$
\begin{aligned}
T_{\left(k_{1}, r_{1}\right)\left(k_{3}, r_{2}\right)}(n)=n \frac{\zeta\left(k_{2}\right)}{\zeta\left(r_{2}\right)} \prod_{p}\left(1+\frac{1-p^{-k_{3}}}{1-p^{-r_{2}}} \cdot \frac{p^{-k_{1}}-p^{-r_{1}}}{1-p^{-r_{1}}}\right) A(n)+ \\
+O\left(n^{\left(r_{1}+r_{2}-2\right) /\left(r_{1} r_{2}-1\right)+\varepsilon}\right)
\end{aligned}
$$

for every $\varepsilon>0$, where the $O$-constant is independent of $k_{1}, k_{2}$ and $n$ :

$$
\begin{aligned}
A(n)=\prod_{p \mid n}\{1+ & \frac{1-p^{-k_{2}}}{1-p^{-r_{2}}}\left(\sum_{\mu=1}^{\infty} p^{-\mu k_{2}} \sum_{m=0}^{\infty}\left[\frac{\varepsilon_{n}\left(\left(p^{m k_{2}}, p^{\mu k_{1}}\right)\right)}{p^{m k_{2}}}-\frac{\left.\varepsilon_{n}\left(\left(p^{r_{2}+m k_{2}}, p^{\mu k_{1}}\right)\right)\right]}{p^{r_{2}+m k_{2}}}\right]-\right. \\
& \left.\left.-\sum_{\mu=0}^{\infty} p^{-\left(r_{1}+\mu k_{1}\right)} \sum_{m=0}^{\infty}\left[\frac{\varepsilon_{n}\left(\left(p^{m k_{2}}, p^{r_{1}+\mu k_{1}}\right)\right)}{p^{m k_{2}}}-\frac{\varepsilon_{n}\left(\left(p^{r_{2}+m k_{2}}, p^{r_{1}+\mu k_{3}}\right)\right)}{p^{r_{3}+m k_{2}}}\right]\right)\right\} \times \\
& \times\left(1+\frac{1-p^{-k_{2}}}{1-p^{-r_{2}}} \cdot \frac{p^{-k_{1}}-p^{-r_{1}}}{1-p^{-k_{1}}}\right)^{-1} .
\end{aligned}
$$

Proof. - It has been shown by M. V. Subba Rao and V. C. Harris (cf. [16], Theorem 3) that

$$
q_{k, r}(m)=\sum_{d ! m} \lambda_{k, r}(d)
$$

where $\lambda_{k, r}$ is the multiplicative function defined by

$$
\lambda_{k, r}\left(p^{\alpha}\right)=\left\{\begin{aligned}
1, & \text { if } \alpha \equiv 0(\bmod k) \\
-1, & \alpha \equiv r(\bmod k) \\
0, & \text { otherwise }
\end{aligned}\right.
$$

Now we take $\Phi_{r_{1}}=Q_{k_{1}, r_{1}}, S_{r_{2}}^{\prime}=Q_{k_{3}, r_{2}}, k=r_{1}$ and $l=r_{2}$ in Theorem 1. Noting that in this case $g_{k}=g_{r_{1}}=\lambda_{k_{1}, r_{1}}$, and $g_{l}^{\prime}=g_{r_{2}}^{\prime}=\lambda_{k_{2}, r_{3}}$, we obtain corollary 4.1 from (4.3) and Theorem 1.

REMARK 4.1. - It has been established by E. Brinitzer (cf. [1], Satz 2) that

$$
\begin{aligned}
T_{\left(k_{1}, r_{1}\right)\left(k_{2}, r_{2}\right)}(n)=n \frac{\zeta\left(k_{2}\right)}{\zeta\left(r_{2}\right)} \prod_{p}\left(1+\frac{1-p^{-k_{2}}}{1-p^{-r_{2}}} \cdot \frac{p^{-k_{1}}-p^{-r_{1}}}{1-p^{-k_{1}}}\right) B(n)+ \\
+O\left(n^{1 / r_{1}+1 / r_{2}-1 / r_{1} r_{2} \zeta} \zeta\left(k_{1} / r_{1}\right) \zeta\left(k_{2} / r_{2}\right)\right)
\end{aligned}
$$


where

$$
\begin{aligned}
& \text { (4.5) } \quad B(n)=\prod_{\mathscr{D} \mid n}\left(1+\frac{1-p^{-k_{2}}}{1-p^{-r_{2}}}\left\{\sum_{\mu=1}^{\infty} p^{-\mu k_{2}} \sum_{m=0}^{\infty}\left[\frac{\left(p^{m k_{2}}, p^{\mu k_{1}}\right)}{p^{m k_{2}}}-\frac{\left(p^{r_{2}+m k_{2}}, p^{\mu k_{1}}\right)}{p^{r_{2}+m k_{2}}}\right]-\right.\right. \\
& \left.\left.-\sum_{\mu=0}^{\infty} p^{-\left(r_{1}+\mu k_{1}\right)} \sum_{m=0}^{\infty}\left[\frac{\left(p^{m k_{2}}, p^{r_{1}+\mu k_{1}}\right)}{p^{m k_{2}}}-\frac{\left(p^{r_{2}+m k_{2}}, p^{\left.r_{1}+\mu k_{2}\right)}\right.}{p^{r_{2}+m k_{2}}}\right]\right\}\right) \times\left(1+\frac{1-p^{-k_{2}}}{1-p^{-r_{2}}} \cdot \frac{p^{-k_{1}}-p^{r_{1}}}{1-p^{-k_{1}}}\right)^{-1} .
\end{aligned}
$$

We remark here that the main term in (4.4) with $B(n)$ given by (4.5) is incorrect. We get the correct result only if we replace each bracket $($,$) by \varepsilon_{n}(()$,$) in the defi-$ nition of $B(n)$ given in (4.5) and then it is clear that the main terms in (4.1) and (4.4) coincide. Further, the error term we obtain from (4.1) is better than the one obtained by E. Brinitzer (cf. [1], Satz 2). It may be mentioned here that M. V. SuBBa RAo and Y.K. FENG [17] established an asymptotic formula for $T_{\left(k_{1}, r_{1}\right)\left(k_{2}, r_{2}\right)}(n)$ in the particular case when $\left(k_{i}, r_{i}\right)=s_{i}>1$ for $i=1,2$ with a $O$-estimate for the error term weaker than that of E. BRINITZER [1].

CoRoLLARY 4.2. - Let $T_{\left(k_{1}, r_{2}\right)\left(k_{2}, r_{2}\right)}^{\prime}(n)$ denote the number of representations of $n$ as the sum of a $\left(k_{1}, r_{1}\right)$-free and $\left(k_{2}, r_{2}\right)$-free integers. Then we have

$$
T_{\left(k_{1}, r_{1}\right)\left(k_{8}, r_{2}\right)}^{\prime}(n)=n \cdot C(n) \prod_{p}\left(1-p^{-r_{1}}-p^{-r_{2}}+p^{-k_{1}}+p^{-k_{2}}\right)+O\left(n^{\left(r_{1}+r_{2}-2\right) /\left(r_{1} r_{2}-1\right)+\varepsilon}\right)
$$

for every $\varepsilon>0$, where the $O$-constant is independent of $k_{1}, k_{2}$ and $n ; C(n)$ being given by

$$
\begin{aligned}
C(n)=\prod_{p r_{1} \mid r_{n}}\left(1-p^{-r_{1}}-p^{-r_{2}}+p^{-k_{1}}+p^{-k_{2}}\right)^{-1} \times \\
\quad \times\left(1-p^{-r_{2}}+p^{-k_{2}}-\varepsilon_{n}\left(\left(p^{k_{2}}, p^{r_{1}}\right)\right) p^{-\left(k_{2}+r_{1}\right)}+\varepsilon_{n}\left(\left(p^{k_{2}}, p^{k_{1}}\right)\right) p^{-\left(k_{1}+k_{2}\right)}\right) .
\end{aligned}
$$

Proof. - First we note that for each prime $p$,

$$
f_{(k, r)}\left(p^{\alpha}\right)=q_{(k, r)}\left(p^{\alpha}\right)-q_{(k, r)}\left(p^{\alpha-1}\right)=\left\{\begin{array}{cc}
-1, & \text { if } \alpha=r \\
1, & \text { if } \alpha=k \\
0, & \text { otherwise }
\end{array}\right.
$$

Now, we take $k=r_{1}, l=r_{2}, S_{k}=Q_{\left(k_{1}, r_{1}\right)}$ and $s_{l}^{\prime}=Q_{\left(k_{2}, r_{2}\right)}$. Taking $k=r_{1}$ and $l=r_{2}$ in (1.1) and (1.2), from (4.8) it is clear that $g_{k}=g_{r_{1}}^{\prime}=f_{\left(k_{1}, r_{1}\right)}$ and $g_{l}^{\prime}=g_{r_{2}}^{\prime}=f_{\left(k_{2}, r_{2}\right)}$. Now, corollary 4.2 can be deduced from (3.9) with the aid of (4.8).

REMARK 4.2. - Formula (4.6) has been established by G. E. HARDY [10] with error term $O\left(n^{1-\delta}\right)$ where

$$
\delta=\operatorname{Min}\left\{1-1 / r_{2}-\varepsilon\left(r_{2}-1\right), 1-1 / r_{1}-1 / r_{2}+\varepsilon\right\}, \quad \text { and } \quad 0<\varepsilon<1 / r_{2} .
$$


It is clear that $1-\delta \geqslant 1 / r_{1}+1 / r_{2}-1 / r_{1} r_{2}$ with equality if and only if $\varepsilon=1 / r_{1} r_{2}$. Thus the best $O$-estimate of the error term that can be obtained from G. E. Hardy's result is $O\left(n^{1 / r_{1}+1 / r_{2}-1 / r_{1} r_{2}}\right)$, which is clearly weaker than the one obtained by us in (4.6).

Letting $k_{1}, k_{2} \rightarrow \infty$ in Corollaries 4.1 or 4.2 , we obtain the important

COROLIARY 4.3. - Let $T_{r_{1}, r_{2}}(n)$ denote the number of ordered pairs $(a, b) \in Q_{r_{1}} \times Q_{r_{3}}$ with $a+b=n$. Then for sufficiently large $n$,

$$
\begin{aligned}
T_{r_{1}, r_{2}}(n)=n \cdot \prod_{D}\left(1-p^{-r_{1}}-p^{-r_{2}}\right) \prod_{\left.p_{r_{1}}\right] n}\left(1+\left(p^{r_{2}}-p^{r_{2}-r_{2}}+1\right)^{-1}\right)+ \\
+O\left(n^{\left(r_{1}+r_{2}-2\right) /\left(r_{1} r_{2}-1\right)+\varepsilon}\right)
\end{aligned}
$$

or every $\varepsilon>0$.

Remark 4.3. - Formula (4.9) is due to A. Page [12]. E. Cohen and R. L. RoBINson (cf. [7], Corollary 2, p. 291) established the asymptotic formula (4.9) without $O$-term. In case $r_{1}=r_{2},(4.9)$ reduces to a formula established originally by C. J. A. EVELYN and E. H. LINFOO' [9].

Coroliary 4.4. - Let $E_{r_{1}, r_{2}}(n)$ denote the error term in the formula (4.9). Then we have the following better $O$-estimates than in (4.9):

$$
E_{r_{1}, r_{3}}(n)=O\left(n^{\left(r_{1}+r_{3}\right) / r_{2}\left(r_{1}+1\right)}(\log n)^{\left.\left(r_{1}^{3} /\left(r_{1}+1\right)-1\right)\left(r_{2}-1\right) / r_{2}\right)} \quad \text { or } O\left(n^{1 / r_{1}}\right)\right.
$$

according as $r_{2} \leqslant r_{1}^{2}$ or $r_{2}>r_{1}^{2}$.

Proof. - Taking $l=r_{2}$ and $S_{l}^{\prime}=Q_{r_{\mathrm{s}}}$ in Theorems 2 and 3 , we obtain Corollary 4.4.

REMARK 4.4. - The result in corollary 4.4 has been recently established by C. PoMERENCE and. D. SURYANARAYAYA [13]. Taking $r_{1}=r_{2}=2$ in corollary 4.4, we obtain that $E_{2,2}(n)=E(n)=O\left(n^{2 / 3}(\log n)^{\left(2^{1 / 3}-1\right) / 2}\right)$ which is an improvement over the result $E(n)=O\left(n^{2 / 3} \log ^{2} n\right)$, established by E. CoHEr [6].

REMARK 4.5. - Taking $k_{1}=r_{1}+1$ and $k_{2}=r_{2}+1$ in (4.6) we obtain an asymptotic formula for $T_{r_{1}, r_{2}}^{8^{*}}(n)$, where $T_{r_{1}, r_{2}}^{s^{*}}(n)$ is the number of representations of $n$ as the sum of a Semi- $r_{1}$-free and a Semi- $r_{2}$-free integer.

CoRoLLARY 4.5. - Let $T_{\left(k_{1}, t_{1}\right)\left(k_{2}, t_{2}\right)}^{*}(n)$ denote the number of ordered pairs $(a, b) \in$ $\in Q_{k_{1}, t_{2}}^{*} \times Q_{k_{2}, t_{2}}^{*}$ with $n=a+b$. Then for sufficiently large $n$ we have

$$
\begin{gathered}
T_{\left(k_{1}, t_{1}\right)\left(k_{2}, t_{2}\right)}^{*}(n)=n \cdot \prod_{p}\left(1-\frac{(p-1)}{p}\left(\frac{p^{k_{1} t_{1}}-1}{p^{k_{1} t_{1}}\left(p^{k_{1}}-1\right)}+\frac{p^{k_{2} t_{2}}-1}{p^{k_{2} t_{2}}\left(p^{k_{2}}-1\right)}\right)\right) \times \\
\times \prod_{p \mid n}\left(1-\frac{(p-1)}{p}\left(\frac{p^{k_{1} t_{1}}-1}{p^{k_{1} t_{1}}\left(p^{k_{1}}-1\right)}+\frac{p^{k_{2} t_{2}}-1}{p^{k_{2} t_{2}}\left(p^{k_{2}}-1\right)}\right)\right)^{-1} \times H^{\prime}(n)+O\left(n^{\left(k_{1}+k_{2}-2\right) /\left(k_{2} k_{2}-1\right)+\varepsilon}\right),
\end{gathered}
$$


for every $\varepsilon>0$, where the $O$-constant is independent of $t_{1}, t_{2}$ and $n ; H^{\prime}(n)$ being given by

$$
\begin{aligned}
H^{\prime}(n)=\prod_{p \mid n}\{1- & \frac{(p-1)}{p}\left(\frac{p^{k_{1} t_{1}}-1}{p^{k_{1} t_{1}}\left(p^{k_{1}}-1\right)}+\frac{p^{k_{3} t_{2}}-1}{p^{k_{8} t_{2}}\left(p^{k_{2}}-1\right)}\right)- \\
& -\sum_{i=1}^{t t_{2}} p^{-t k_{2}}\left[\sum_{j=1}^{t_{1}}\left(\frac{\varepsilon_{n}\left(\left(p^{t k_{2}}, p^{j k_{1}+1}\right)\right)}{p^{j k_{1}+1}}-\frac{\varepsilon_{n}\left(\left(p^{t k_{2}}, p^{j k_{2}}\right)\right)}{p^{j k_{1}}}\right)\right]- \\
& \left.-\sum_{i=1}^{t_{2}} p^{-\left(t k_{2}+1\right)}\left[\sum_{j=1}^{t_{1}}\left(\frac{\varepsilon_{n}\left(\left(p^{t k_{2}+1}, p^{j k_{1}+1}\right)\right)}{p^{j k_{1}+1}}-\frac{\varepsilon_{n}\left(\left(p^{i k_{2}+1}, p^{j k_{2}}\right)\right)}{p^{j k_{1}}}\right)\right]\right\} .
\end{aligned}
$$

Proof. - It can be shown that

$$
f_{k, t}^{*}\left(p^{\alpha}\right)=q_{k, t}^{*}\left(p^{\alpha}\right)-q_{k, t}^{*}\left(p^{\alpha-1}\right)=\left\{\begin{aligned}
-1, & \text { if } \alpha=j k, 1 \leqslant j \leqslant t \\
1, & \text { if } \alpha=j k+1,1 \leqslant j \leqslant t \\
0, & \text { otherwise. }
\end{aligned}\right.
$$

Now we take $k=k_{1}, l=k_{2}, S_{k}=Q_{k_{1}, t_{1}}^{*}$ and $S_{l}^{\prime}=Q_{k_{2}, t_{2}}^{*}$. Then it is clear that $g_{k_{1}}=$ $=f_{k_{1}, t_{1}}^{*}$ and $g_{k_{\mathrm{a}}}=f_{k_{2}, t_{\mathrm{a}}}^{*}$.

Now, Corollary 4.5 can be deduced from (3.9) with the aid of (4.12).

REMARK 4.6. - Letting $t_{1}, t_{2} \rightarrow \infty$ in (4.10) we obtain an asymptotic formula for $T_{k_{1}, k_{2}}^{*}(n)$, where $T_{k_{1}, k_{2}}^{*}(n)$ denotes the number of representations of $n$ as the sum of a unitarily $k_{1}$-free integer and a unitarily $k_{2}$-free integer.

COROLLARY 4.6. - Let $T_{\left(r_{2}, t_{2}\right) r_{1}}^{*}(n)$ denote the number of ordered pairs $(a, b) \in$ $\in Q_{r_{2}, t_{2}}^{*} \times Q_{r_{1}}$ with $n=a+b$. Then for sufficiently large $n$ we have

$$
\begin{aligned}
T_{\left(r_{2}, t_{2}\right) r_{1}}^{*}(n)=n \cdot \prod_{p}\{1- & \left.\frac{1}{p^{r_{1}}}-\frac{1}{p^{r_{3} t_{2}+1}}\left(\frac{p^{r_{2} t_{2}}-1}{p^{r_{3}}-1}\right)\right\} \times \\
& \times \prod_{p r_{1}, n}\left(1-\frac{p^{r_{1}-1-r_{2} t_{2}}(p-1)\left(p^{r_{2} t_{2}}-1\right)}{\left(p^{r_{1}}-1\right)\left(p^{r_{2}}-1\right)}\right)^{-1}+E_{r_{1}, r_{2}}^{\left(t_{2}\right)}(n),
\end{aligned}
$$

where

$$
W_{r_{1}, r_{2}}^{\left(t_{3}\right)}(n)=O\left(n^{\left(r_{1}+r_{3}\right) / r_{3}\left(r_{1}+1\right)}(\log n)^{\left(r_{1}^{1} /\left(r_{1}+1\right)-1\right)\left(r_{3}-1\right) / r_{2}}\right) \quad \text { or } O\left(n^{1 / r_{2}}\right),
$$

according as $r_{2} \leqslant r_{1}^{2}$ or $r_{2}>r_{1}^{2}$; the $O$-estimates being independent of $t_{2}$ and $n$.

Proof. - Taking $k=r_{1}, l=r_{2}, S_{l}^{\prime}=Q_{r_{2}, t_{2}}^{*}$ in Theorems 2 and 3 , and using (4.12) $\left(k=r_{2}, t=t_{2}\right)$ we obtain Corollary 4.6.

Let $t \geqslant 3$ and $s_{t}^{*}$ denote the set introduced in the beginning of section 4 . Now, we have

COROLLARY 4.7. - Let $T_{t}^{*}(n)$ denote the number of ordered pairs $(a, b) \in S_{t}^{*} \times Q_{2}$ 
with $n=a+b$. Then for sufficiently large $n$,

$$
\begin{aligned}
T_{t}^{*}(n)=n & \prod_{p}\left(1-2 p^{-2}+p^{-t}-p^{-(t+1)}\right) \cdot \\
& \cdot \prod_{p^{2} \mid p n}\left(1+\frac{p^{2}}{p^{2}-1}\left(p^{-t}-p^{-(t+1)}-p^{-2}\right)\right)^{-1}+O\left(n^{2 / 3}(\log n)^{\left(2^{1 / 3}-1\right) / 2}\right),
\end{aligned}
$$

where the $O$-estimate is independent of $t$ and $n$.

Proof. - Since $Q_{2} \subseteq S_{t}^{*}$, taking $k=l=2$ and $S_{2}=S_{t}^{*}$ in Theorem 3, we obtain (4.14).

Added in proof. - We note that Theorem 1 of this paper enables us to obtain an asymptotic formula for $T_{0}(n)$, where $T_{0}(n)$ denotes the number of representations of $n$ in the form $n=a+b$, where $a$ is a $K_{1}$-void integer and $b$ is a $K_{2}$-void integer. For the definition of $K$-void integers we refer to G. J. RIEGER (J. reine angew. Math., 262/263 (1973), pp. 189-193).

\section{REFERENCES}

[1] E. BrinitzeR, Über $(k, r)$-Zahlen, Monatsh. Math., 80 (1975), pp. 31-35.

[2] L. Carlitz, On a problem in additive arithmetic, Quart. J. Math. (Oxford), 3 (1932), pp. 273-290.

[3] E. Cohen, Some sets of integers related to the 7-free integers, Acta Sci. Math. (Szeged), 22 (1961), pp. 223-233.

[4] E. CoHes, Arithmetical Notes III, certain equally distributed sets of integers, Pacific J. Math., 12 (1962), pp. 77-84.

[5] E. CoHEN, Arithmetical Notes XIII, A sequal to note IV, Ele. Math., 18 (1963), pp. 8.11.

[6] E. CoHEN, The number of representations of an integer as a sum of two square-free numbers, Duke Math. J., 32 (1965), pp. 181-185.

[7] E. COHEN - R. L. RoBIsson, On the distribution of the k-free integers in residue classes, Acta Arith., 8 (1962-63), pp. 283-293; Errata, ibid., 10 (1964-65), p. 443; Correction, ibid., $16(1969-70)$, p. 439.

[8] T. Estermarn, On the representation of a number as a sum of two numbers not divisible by $k$-th powers, J. London Math. Soc., 6 (1931), pp. 37-40.

[9] C. J. A. EVELYN - E. H. Linfoot, On a problem in the additive theory of numbers (second paper), J. Reine. Angew. Math., 164 (1931), pp. 131-140.

[10] G. E. HARDY, The distribution of $(k, r)$-free integers in residue classes, Notices Amer. Math. Soc., 23 (1976), p. A.53.

[11] G. H. HARDY - E. M. WRIGHT, An introduction to the theory of numbers, 4th ed., Calender press, Oxford, 1960.

[12] A. PAGE, An asymptotic formula in the theory of numbers, J. London Math. Soc. (1932), pp. 24-27.

[13] C. Pomerance - D. Suryanarayana, On a problem of Evelyn-Linjoot and Page in additive number theory, Publicationes Mathematicae, Debrecen, 26 (1979), pp. 237-244.

[14] K. PrachaR, Über die kleinste quadratfreie Zahl einer arithmetischen Reihe, Monatsh. Math., 62 (1958), pp. 173-176. 
[15] V. SitA RAMAIAII - D. SURYANARAYANA, The number of representations of an integer as the sum of a prime and an element in a set, Bollettino U.M.I. (to appear).

[16] M. V. Subbarao - V. C. HaRRis, A new generalization of Ramanujan's sum, J. London Math. Soc., 41 (1966), pp. 595-604.

[17] M. V. SUbBaRaO - Y. K. FENG, On the distribution of generalized k-free integers in residue classes, Duke Math. J., 38 (1971), pp. 741-748.

[18] D. Suryanarayana, Semi-k-free integers, Ele. Math., 26 (1971), pp. 39-40.

[19] B. M. WILson, Proofs of some formulae enuntiated by Ramanujan, Proc. London Math. Soc., (2) 24 (1922), pp, 235-255. 